\title{
Identificación de sujetos a riesgo de deficiencia de hierromediante el Indicé Receptor Soluble de Transferrina-Log Ferritina sérica en hombres afrodescendientes residentes en San Basilio de Palenque y Cartagena de Indias, DTyC., Bolívar, Colombia.
}

\author{
Martha Castillo Bohórquez ${ }^{1}$, Ana Isabel Mora Bautista ${ }^{1}$ \\ Katherine Laiton Donato², Francy Pérez Llanos², \\ Milena Tapiero Rodríguez ${ }^{2}$
}

\begin{abstract}
Bacterióloga Mag. Docente Investigadora, Grupo Eritrón, Universidad Colegio Mayor de Cundinamarca. Estudiante Programa de Bacteriología y Laboratorio Clínico de la Universidad Colegio Mayor de Cundinamarca.
\end{abstract}

Correspondencia: aimora@unicolmayor.edu.co

Recibido: 02-10-10/ Aceptado: 05-15-10

\section{Resumen}

En el ámbito mundial, sin distingo de raza, edad, género ni procedencia geográfica, la anemia de mayor prevalencia es la anemia ferropénica. De la misma forma, son insuficientes los reportes nacionales e internacionales sobre la prevalencia de los estados subclínicos de hierro, especialmente en comunidad afrodescendiente. El objetivo de la investigación fue caracterizar una población masculina afrodescendiente de 73 hombres sanos con edades comprendidas entre los 16 y 30 años mediante el comportamiento del índice (sTfR- Log F.S.), residentes en San Basilio de Palenque y en Cartagena de Indias DTyC. Se determinaron los índices primarios eritroides: hemoglobina (Hb), hematocrito (Hto), estudio de sangre periférica (E.S.P.); el receptor soluble de transferrina (sTfR), la ferritina sérica (F.S.) y el índice receptor soluble de transferrina - Logaritmo de la ferritina sérica (sTfR-Log F.S.) El análisis estadístico se realizó mediante el software de SPSS versión 17,0.

En la población de San Basilio de Palenque el hallazgo hematológico por el algoritmo de mayor frecuencia se asoció a la enfermedad crónica acompañada de deficiencia de hierro en el 41.6\%, seguido de deficiencia subclínica de hierro estadio II con un 33.3\%. En Cartagena el hallazgo de mayor frecuencia fue la enfermedad crónica con deficiencia de hierro en 49\%, seguido de deficiencia subclínica de hierro estadio || 20.4 I\%. Las dos poblaciones evidenciaron un comportamiento hematológico similar en las diferentes variables, constituyéndose estos resultados en pioneros para futuras investigaciones de los estadios subclínicos que anteceden la deficiencia de hierro en afrodescendientes colombianos.

Palabras clave: Indicé Receptor Soluble de Transferrina- Log Ferritina Sérica, deficiencia subclínica de hierro, afrodescendientes, anemia ferropénica, anemia por enfermedad crónica. 


\section{Abstract \\ Identification of iron deficiency risk by index soluble transferrin receptor-log ferritin in African descent men living at San Basilio de Palenque, Cartagena de Indias, DT and C., Bolivar, Colombia.}

Worldwide, iron deficiency anemia is the most prevalence anemia, regardless of race, age, gender or geographical origin; alike there is insufficient the data and reports on the prevalence of subclinical iron states especially in the African descendant community national and internationally. The research aimed to characterize a healthy male population of African descendent aged between 16 and 30 years through the index (sTfR-Log FS) in 73 African descent men living in San Basilio de Palenque and Cartagena de Indias DT y C. Erythroid index were determined: hemoglobin $(\mathrm{Hb})$, hematocrit $(\mathrm{HCT})$, peripheral blood smear evaluation, the soluble transferrin receptor (sTfR), serum ferritin (SF) and soluble transferrin receptor index - Logarithm of serum ferritin (sTfR-Log FS). Statistical analysis was performed using SPSS software version 17.0.

Using the sTfR-Log FS, at San Basilio de Palenque, the hematologic finding by algorithm is most often associated with chronic disease along with iron deficiency (41.6\%), followed by subclinical iron deficiency stage II (33.3\%). In Cartagena, chronic iron deficiency (49\%) was the most frequent found it, followed by subclinical iron deficiency stage II (20.4 I\%). The two populations showed similar behavior in different hematologic variables, these results becoming pioneers for future research of the subclinical stages preceding iron deficiency in Colombia's Afro-descendants.

Keywords: soluble transferrin receptor index-Log serum ferritin, subclinical deficiency of iron, African descendant, ferropenic anemia, anemia of chronic disease.

\section{Introducción}

La prevalencia de anemia ferropénica en el ámbito mundial es del 48.8\%, según los datos suministrados por la Organización Mundial de la Salud (OMS); en población latinoamericana la prevalencia es del 58\%; se estima que Colombia tiene una prevalencia entre el 20\% y 39.9\% de la población' Antes de desarrollar anemia ferropénica se presentan deficiencias subclínicas de hierro, estas deficiencia pueden cursar en estadio I con una depleción progresiva del hierro de los depósitos con eritropoyesis normal y sin alteración morfológica en el extendido de sangre (1), el estadio II presenta depleción del compartimiento de depósito de hierro, mientras se utiliza el hierro disponible en el compartimiento de transporte la producción de eritrocitos continua normal y pueden afectarse otros tejidos dependientes de hierro como los músculos con síntomas inespecíficos(1) Por último en el estadio III se manifiesta la anemia ferropénica en el que la hemoglobina se encuentra por debajo de los valores de referencia ante la depleción de los depósitos y se presenta disminución de hierro de transporte. En esta fase los pacientes experimentan los síntomas de la anemia los cuales son fatiga, debilidad y palidez mucocutánea (2).

El diagnóstico de deficiencia subclínica de hierro no se logra con análisis hematológicos de rutina; determinación de hemoglobina y hematocrito, por lo que actualmente se incorpora la determinación del sTfR combinado con el sTfR-Log F.S., prueba capaz de informar sobre el almacenamiento y el compartimiento funcional del hierro con una sensibilidad del 92-94\% y especificidad del 98\% (1). El diagnóstico de deficiencias subclínicas de hierro en pacientes afrodescendientes puede ser difícil, debido a que algunos parámetros hematológicos están por debajo de los valores de referencia. Algunos estudios plantean que la hemoglobina se encuentra disminuida posiblemente por los niveles de 2,3-difosfoglicerato que son mayores en esta raza y se correlacionan inversamente con la hemoglobina. Otra hipótesis 
indica que aproximadamente $1,4 \%$ de la población afrodescendiente colombiana presenta talasemias de rasgo heterocigoto (3). Por otro lado se contempla la disminución de la hemoglobina con el rasgo de célula falciforme, que se produce en aproximadamente el $8 \%$ de la población y la deficiencia de glucosa 6-fosfato deshidrogenasa (4).

Las personas afrodescendientes presentan características hematológicas propias de su raza, debido a numerosos cambios adaptativos tanto genéticos como ambientales (4), a esto se suman los bajos aportes nutricionales de hierro que puede causar reacciones adversas sobre su salud (5). Esta investigación tuvo como finalidad caracterizar hematológicamente a una población afrodescendiente colombiana teniendo como base estudios realizados en poblaciones africanas que presentan características hematológicas propias de su raza y demostrando que el sTfr-Log F.S es una prueba aplicable a distintas poblaciones.

\section{Materiales y métodos}

Selección de la muestra. Se realizó extracción sanguínea venosa de doble tubo tapa lila y tapa roja en 73 hombres afrodescendientes sanos con edades comprendidas entre 16 y 30 años. Del total de las muestras 24 pertenecían a hombres afrodescendientes de San Basilio de Palenque (Bolívar, Colombia) escolarizados, obtenidas con previo consentimiento informado, encuesta sociodemográfica de salud y estado nutricional con ayuno de 8 horas. Las 49 muestras restantes procedían de hombres afrodescendientes habitantes en Cartagena de Indias D.T.yC., obtenidas por muestreo ciego en el Hospital Naval de Cartagena. Los 73 hombres presentaron índices primarios eritroides dentro de los valores de variabilidad biológica.

Determinaciones hematológicas. La Hemoglobina y Hematocrito (Hb-Hto) se determinaron en San Basilio de Palenque mediante el Hemoglobinómetro "Hbmeter" de Human y por microcentrífuga respectivamente. En Cartagena de Indias D.T.yC., mediante hemoanalizador de IV Generación, como criterio de exclusión para este parámetro se tomó la concentración de hemoglobina por debajo de $13 \mathrm{~g} / \mathrm{dL}$.
Receptor soluble de transferrina ( $s$ TfR). Determinado mediante el ensayo inmunológico basado en la técnica ELISA sándwich (Quantikine, IVD. Human sTfRImmunoassayRef DTFR1 R\&D Systems). El sistema utiliza un anticuerpo monoclonal anti-sTfR inmovilizado en la microplaca; la cantidad de conjugado que permanece en la celda es proporcional a la cantidad de sTfR capturado. Los valores de referencia para sujetos de raza negra y residentes de alturas iguales o superiores a $1600 \mathrm{msnm}$ están incrementados en un $6 \%$ en el valor superior tal como se referencia entre 8.7 y $29.8 \mathrm{nmol} / \mathrm{L} \mathrm{(6-7).}$

Ferritina sérica (F.S.). Se realizó mediante el ensayo inmunológico basado en la técnica ELISA sándwich (Diagnostic Automation INC. Microwell ELISA Human Ferritin Enzyme Immunoassay Test Kit Ferritin Cat \# 1601 Z).Los valores de referencia para hombres tomados en este estudios corresponden al rango entre $32-501 \mathrm{ng} / \mathrm{mL}$.

Índice receptor soluble de transferrina / Log ferritina sérica. La determinación del índice sTfRFS se obtuvo a partir de los valores de FS y del sTfR de cada muestra, los cuales se asociaron de acuerdo a la siguiente fórmula propuesta por Skikne: sTfR/ $\log$ FS (7-9). El sTfR-FS se obtuvo dividiendo el RsTf, expresado en $\mathrm{mg} / \mathrm{L}$ entre el logaritmo en base 10 de la ferritina sérica. En sujetos que viven a nivel del mar, sin enfermedades inflamatorias crónicas ni neoplásicas, los valores mayores a 1,5 indican deficiencias de hierro.

Proteína C Reactiva. Mediante técnica convencional de aglutinación para descartar proceso infeccioso o inflamatorio crónico (10).

Análisis estadístico. Se evaluó la distribución de normalidad: utilizando la prueba de Shapiro-Wilk (criterio de normalidad: $\mathrm{p} \geq 0.05$ ). Con base en el resultado de esta prueba se describieron las medidas de tendencia central: promedio o mediana y las medidas de dispersión: desviación estándar (SD) o los rangos mínimo y máximo. Se establecieron proporciones para la descripción de la variable categórica. Para establecer la diferencia de los indicadores hematológicos entre las dos poblaciones participantes, primero se evaluó la homogeneidad de las varianzas de las 
Tabla 1. Descripción de las características de los hombres afrodescendientes de San Basilio de Palenque y Cartagena de Indias, D.T. y C.

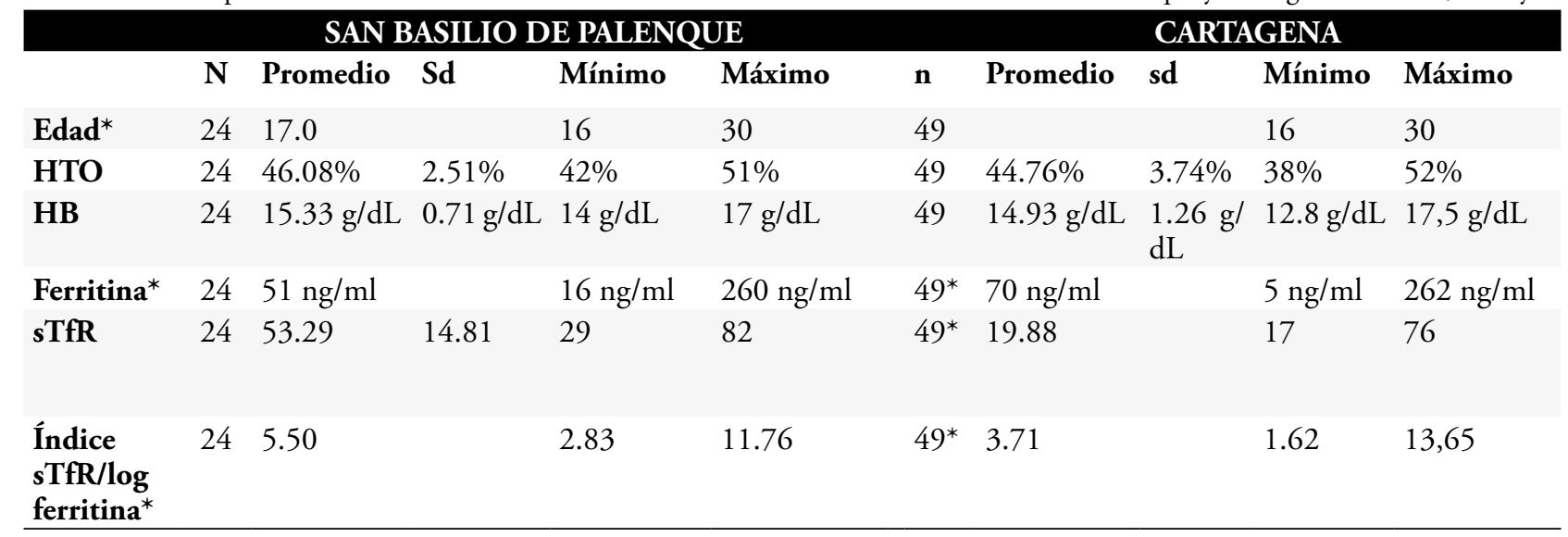

* Mediana en San Basilio de Palenque y Cartagena

NA: no aplica

variables numéricas, para esto se usó la prueba de Levene (no homogeneidad de las varianzas: $\mathrm{p} \leq 0.05$ ). Si la variable cumplía con el criterio de normalidad y de homogeneidad de las varianzas o el de normalidad se utilizó la prueba "t de Student", y la prueba de U de Mann-Whitney si no cumplía el criterio de normalidad; se consideró una prueba significativa a dos colas con una $\mathrm{p} \leq 0.05$. La prueba Ji al cuadrado de diferencias se utilizó para valorar las diferencias de proporciones de los hallazgos hematológicos entre las dos poblaciones con un valor significativo de $\mathrm{p} \leq 0.05$. También se valoró la correlación entre la ferritina y el receptor soluble de transferrina para cada grupo poblacional participante a través de la prueba de Pearson, significativa con un valor de $\mathrm{p} \leq 0.05$. Se utilizó el programa estadístico SPSS versión 17.0.

\section{Resultados}

De 61hombres afrodescendientes escolarizados residentes en San Basilio de Palenque pertenecientes a la comunidad y asistentes a la Institución Educativa Benkos Biohó, el 83.6\% son residentes de San Basilio de Palenque desde hace más de 10 años (nativos); el $37.7 \%$ se encuentra en el régimen vinculado del sistema general de salud; el $63.3 \%$ no cuenta con cobertura del sistema de salud y el $93.4 \%$ cuenta con el servicio de acueducto y agua potable.

El 90\% de los encuestados manifestaron un estado de salud excelente o buena, el 55\% recibió la última valoración médica de 2 a 6 meses y el 95\% no ha sido donante de sangre en los últimos tres meses.
La enfermedad infecciosa que se presentó con el mayor número de casos fue la infección respiratoria aguda (IRA) con una frecuencia del 18.3\% de casos, seguida de enfermedad diarreica aguda (EDA) con $16.6 \%$ de casos. Se destaca la presencia de eosinofilia en la población con asociación aparente a poliparasitismo, síndrome anémico en el 8.3\%, sangrados y desnutrición en el 1.6\% de la población.

De los 61 encuestados el $60 \%$ no fue incluido en el estudio por inasistencia, falta de cumplimiento en requisitos previos ó formar parte del grupo de los criterios de exclusión. Estos hallazgos están en contraposición con la población estudiada cartagenera quién goza de mejores condiciones salubres y sociodemográficas

Entre los dos grupos seleccionados, se analizaron un total de 73 muestras. En la ciudad de Cartagena las variables edad, F.S, sTfR y sTfR/log F.S. no siguieron una distribución normal como se demostró en la prueba estadística Shapiro-Wilk: $\mathrm{p} \leq 0.05$, mientras que en San Basilio de Palenque los participantes siguieron una distribución normal, Shapiro-Wilk: p> 0.05. Así mismo, el Hto, la $\mathrm{Hb}$ y el sTfR siguieron una distribución normal en ambas poblaciones, Shapiro-WilK $\mathrm{p} \geq 0.05$, Tabla 1 .

El hallazgo de mayor frecuencia fue la anemia por enfermedad crónica con deficiencia de hierro con un $49 \%$ seguido de deficiencia subclínica de hierro estadio II 20.41\%, Figura No 1.

Análisis comparativo de los indicadores biológicos entre los dos grupos poblacionales. 


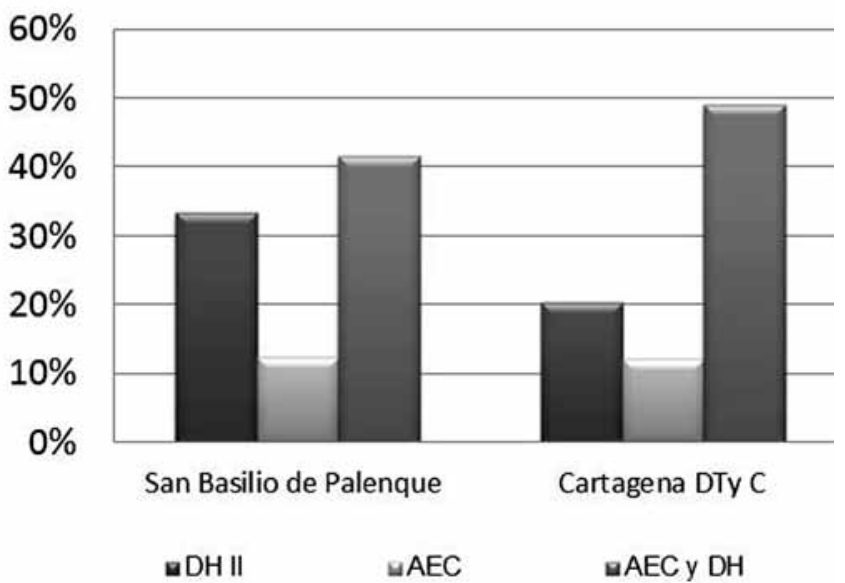

Figura 1. Distribución de los hallazgos hematológicos en los hombres de San Basilio de Palenque y Cartagena de Indias, D.T. y C, Bolívar, Colombia.

DH II: deficiencia subclínica de hierro estadio II.

AEC: anemia por enfermedad crónica.

AEC y DH II: anemia por enfermedad crónica con deficiencia de hierro

Todos los indicadores biológicos presentaron homogeneidad de las varianzas $(\mathrm{p} \geq 0.05)$. El análisis comparativo de los indicadores biológicos entre los hombres de las dos poblaciones estudiadas se presentan en la Tabla 2, se observa que no hubo diferencias en los valores promedio de Hto, $\mathrm{Hb}$ y ferritina en las dos poblaciones ( $\mathrm{p} \geq 0.05)$.

Las medianas de sTfR y el índice sTfR/ log FS fueron mayores en los hombres de San Basilio de Palenque en comparación con los de Cartagena $(\mathrm{p} \leq 0.01)$. Con base en los hallazgos hematológicos se observó que no hubo diferencias entre estos en las dos poblaciones estudiadas ( $\mathrm{p} \geq 0.05)$.

En relación con los valores estándar establecidos para cada uno de los indicadores hematológicos y los índices en las dos poblaciones se observó que en San Basilio de Palenque todos los hombres presentaron valores de Hto iguales o superiores a 42\%. Los niveles de $\mathrm{Hb}$ fueron superiores a $12.5 \mathrm{~g} / \mathrm{dL}$ en todos los hombres; el 33.3\% de los hombres tenían la ferritina menor de $30 \mathrm{ng} / \mathrm{mL}$, el $41.6 \%$ valores entre $30 \mathrm{ng} / \mathrm{mL}$ a $150 \mathrm{ng} / \mathrm{mL}$ y $16.7 \%$ valores superiores a $150 \mathrm{ng} /$ mL. Se halló un sTfR superior a 29.8 en $95.8 \%$ de los hombres. Todos los hombres participantes de San Basilio de Palenque tuvieron valores del índice sTfR/ $\log \mathrm{FS}$ mayores a 2.2 .

Por otra parte, en Cartagena el 16\% de los hombres obtuvo un valor inferior de Hto a $42 \%$. En los niveles de F.S., el $20.4 \%$ presentaron valores menores de $30 \mathrm{ng} / \mathrm{mL}$; el $49 \%$ presentaron valores entre $30 \mathrm{y}$ $150 \mathrm{ng} / \mathrm{mL}$, y el $12.2 \%$ valores superiores a $150 \mathrm{ng} /$ $\mathrm{mL}$. El sTfR mostró valores superiores a $29.8 \mathrm{nmol} / \mathrm{L}$ en el $75,5 \%$ de los hombres afrodescendientes. El $89.8 \%$ de los hombres presentaron valores del índice sTfR/log FS superiores a 2.2 y el $10.2 \%$ presentaron valores mayores a $1.8-2.2$.

$\mathrm{Si}$ se tiene como valor de referencia para el sTfR el rango comprendido entre $8.7-29.8 \mathrm{nmol} / \mathrm{L}$ como hallazgo significativo, se encontró que el $80.8 \%$ de los hombres afrodescendientes sobre-expresaron el receptor por encima del valor de referencia, de tal manera que el $20.5 \%$ lo duplica por encima de 60 $\mathrm{nmol} / \mathrm{L}$ y el $1.5 \%$ lo triplica.

Análisis de la ferritina sérica y el índice receptor soluble de transferrina-ferritinacon los hallazgos hematológicos. En la población de San Basilio de Palenque se encontró que el $41.6 \%$ de los pobladores tenían una ferritina entre $30 \mathrm{ng} / \mathrm{mL}$ a $150 \mathrm{ng} / \mathrm{mL}$ con un índice sTfR/log FS mayor ó igual a 1.8; seguido del $33 \%$ con una ferritina menor a $30 \mathrm{ng} / \mathrm{mL}$ y con un índice sTfR/log FS mayor a 2.2, Tabla 3. Los

Tabla 2. Diferencias en los indicadores hematológicos e índice entre las dos poblaciones afrodescendientes estudiadas.

\begin{tabular}{|c|c|c|c|}
\hline & Diferencia de unidades & t student o Mann-Whitney U & $\mathbf{p}$ \\
\hline Hto & 1.32 & 1.44 & 0.155 \\
\hline $\mathrm{Hb}$ & 0.334 & 1.07 & 0.29 \\
\hline Ferritina* & -10.69 & 523.0 & 0.445 \\
\hline$s \operatorname{TfR}^{*}$ & 14.49 & 271.0 & $<0.001$ \\
\hline $\begin{array}{l}\text { Índice sTfR/log } \\
\text { ferritina* }\end{array}$ & 1.59 & 299.0 & 0.001 \\
\hline
\end{tabular}

*Mann-Whitney U. 
Tabla 3. Hallazgos hematológicos con base en los niveles de ferritina sérica y el índice receptor soluble transferrina- ferritina sérica en la población de San Basilio de Palenque.

\begin{tabular}{|c|c|c|c|c|}
\hline Ferritina Sérica & sTfR/ Log F.S. & $\mathbf{n}$ & $\%$ & Hallazgo compatible \\
\hline$<30 \mathrm{ng} / \mathrm{mL}$ & $>1.8-2.2$ & 0 & 0.0 & Deficiencia hierro I \\
\hline$<30 \mathrm{ng} / \mathrm{mL}$ & $>2.2$ & 8 & 33.3 & Deficiencia subclínica de hierro estadio II \\
\hline $30-150 \mathrm{ng} / \mathrm{mL}$ & $\geq 1.8$ & 10 & 41.6 & $\begin{array}{l}\text { Anemia por enfermedad crónica y } \\
\text { deficiencia de hierro }\end{array}$ \\
\hline$>150-<260 \mathrm{ng} / \mathrm{mL}$ & $>2.2$ & 3 & 12.5 & Anemia por enfermedad crónica \\
\hline \multirow[t]{2}{*}{$\geq 260 \mathrm{ng} / \mathrm{mL}$} & $>2.2$ & 1 & 4.2 & Anemia por enfermedad crónica \\
\hline & & 2 & 8.3 & Normalidad hematológica \\
\hline Total & & 24 & 100 & \\
\hline
\end{tabular}

Tabla 4. Hallazgos hematológicos con base en los niveles de ferritina sérica y el Índice receptor soluble transferrina-ferritina con deficiencia subclínica de hierro en la población de Cartagena D.T.y C.

\begin{tabular}{lllll} 
Ferritina sérica & $\mathbf{s}$ TfR/log FS & $\mathbf{n}$ & $\%$ & Hallazgo compatible \\
& $\leq 1.8$ & 0 & 0.0 & Normal \\
$<\mathbf{3 0} \mathbf{~ n g} / \mathbf{m L}$ & $>1.8-2.2$ & 0 & 0.0 & Deficiencia subclínica de hierro estadio I \\
$<\mathbf{3 0} \mathbf{~ n g} / \mathbf{m L}$ & $>2.2$ & 10 & 20.41 & Deficiencia subclínica de hierro estadio II \\
$\mathbf{3 0 - 1 5 0} \mathbf{~ n g} / \mathbf{m L}$ & $\geq 1.8$ & 24 & 49 & Anemia por enfermedad crónica y deficiencia de hierro \\
$>\mathbf{1 5 0}-\mathbf{2 6 0} \mathbf{~ n g} / \mathbf{m L}$ & $\geq 1.8$ & 6 & 12.2 & Anemia por enfermedad crónica \\
& & 9 & 18.3 & Normalidad hematológica \\
Total & & $\mathbf{4 9}$ & $\mathbf{1 0 0}$ & \\
\hline
\end{tabular}

hombres afrodescendientes de Cartagena presentaron en un $49 \%$ una ferritina entre $30 \mathrm{ng} / \mathrm{mL}$ a $150 \mathrm{ng} / \mathrm{mL}$ con un índice mayor ó igual a 1.8; seguido de $20.4 \%$ de los hombres con una ferritina menor a $30 \mathrm{ng} / \mathrm{ml}$ y con un índice mayor a 2.2, Tabla 4.

\section{Discusión}

Las pruebas de laboratorio comúnmente utilizadas para detectar las deficiencias de hierro pueden generar resultados falsos -negativos en diferentes alturas sobre el nivel del mar, por lo tanto este estudio describió el comportamiento del índice (sTfR-FS)en hombressanos afrodescendientes con edades comprendidas entre los 16 y 35 años nativos y residentes de bajas alturas, para lo cual se emplearon los índices hematológicos primarios y las pruebas bioquímicas FS y sTfR, en este estudio no se encontraron diferencias significativas de la variable altura por debajo de los $1800 \mathrm{msnm}$.

Es importante resaltar que los valores de hemoglobina no deben ser utilizados como herramienta diagnóstica en el síndrome anémico, ni de los estados subclínicos puesto que valores altos pueden estar enmascarando la deficiencia, a diferencia de los receptores solubles de transferrina que aumentan su valor por estímulo eritropoyéticoindicando que hay presencia de $\mathrm{DH}$ por la elevación de sus valores aun cuando la anemia esté ausente; es decir, que el receptor de transferrina tampoco debe usarse solo como indicador de $\mathrm{DH}$, sino en complemento con otras pruebas diagnósticas como la ferritina sérica. Es importante puntualizar que la ferritina sérica es 
considerada reactante de fase aguda, traducida en valores incrementados como respuesta al proceso infeccioso o inflamatorio, lo cual puede enmascarar estadios subclínicos.

Por otra parte, la determinación del índice sTfR- Log FS es una técnica sencilla y de gran valor diagnóstico por especificidad y sensibilidad, siendo de gran utilidad como marcador de deficiencia subclínica de hierro. Sin embargo, es relevante conocer la altitud sobre el nivel del mar y el comportamiento hematológico de la raza de cada población ya que puede tener importantes modificaciones en los valores de referencia, como es el caso de la población afrodescendiente que presenta un aumento del 6\% en el valor del sTfR.

Con respecto a la medición de la ferritina sérica, prueba especializada de tamizaje para depleción de hierro que evalúa los depósitos del biometal, se observó que el $24.6 \%$ de los hombres afrodescendientes estudiados presentaron valores por debajo del límite establecido, a este respecto el punto de corte establecido por la OMS, señala que aquellos valores $\leq 12$ $\mathrm{ng} / \mathrm{mL}$, deben ser considerados deficiencias de hierro.

Como hallazgo importante, el 59\% denotó hallazgos de reactividad para enfermedad, procesos infecciosos ó inflamatorios crónicos, evidenciados por el índice del receptor soluble de transferrinaferritina sérica.

De la misma forma, según reportes previos en otros países (11) la población afrodescendiente evidencia valores de hemoglobina más bajos con relación a la raza blanca, sin embargo en este estudio se evidenció una media de $15.1 \mathrm{~g} / \mathrm{dL}$ para hemoglobina posiblemente atribuible al peso y la talla de los participantes, con un mínimo de $12.8 \mathrm{~g} / \mathrm{dL}$ y un máximo de $17.3 \mathrm{~g} /$ dL. Tampoco se presentaron valores por encima de los valores de referencia de ferritina sérica como también se ha reportado para esta raza (12). Respecto al sTfR en el análisis estadístico se tuvo en cuenta un aumento del valor de referencia superior en un $6 \%$ para la población afrodescendiente.

En el análisis comparativo de los indicadores hematológicos, los dos grupos tienen un comportamiento similar; aun cuando los afrodescendientes de San Basilio de Palenque han conservado los rasgos genéticos de un $82 \%-92 \%$ (13); mientras que los afrodescendientes cartageneros han tenido mayor mestizaje.

El estudio reveló que en el 25\% de la muestra existe deficiencia subclínica de hierro estadio II valorados previamente con el indicador de concentración de hemoglobina bajo criterios de normalidad y sin ningún tipo de hallazgo morfológico al estudio de sangre periférica. Sin embargo los resultados del sTfR indican depleción del compartimiento de depósito de hierro y aumento significativo de ellos en un intento por captar hierro (2) Se considera que la población es susceptible a desarrollar en el corto plazo anemia ferropénica debido a que este estadio subclínico antecede la depleción.

Es importante mencionar, que en la población de San Basilio de Palenque se excluyeron aquellas personas que en la encuesta de salud refirieron haber sido diagnosticados con parasitismo intestinal, anemia ferropénica, enfermedad inflamatoria o infecciosa con tres meses de anterioridad. En ambos grupos el hallazgo hematológico que prevalece fue compatible con anemia secundaria a enfermedad crónica acompańada de deficiencia de hierro en un 46.5\%; criterio diagnóstico que se cumple según el algoritmo diagnóstico de Suominen (8) que explica que cuando el valor de la ferritina es mayor de $30 \mathrm{ng} /$ $\mathrm{mL}$ y el valor del índice sTfR- Log FS es mayor de 1.8 se describe como anemia por enfermedad crónica y deficiencia de hierro (8)debido al enmascaramiento de la deficiencia de hierro por el aumento de la ferritina sérica a pesar de una PCR negativa.

Así mismo, son escasos los estudios que usan el índice sTfR- Log FS para el diagnóstico de deficiencia subclínica de hierro y es la primera vez que se tienen registros de la misma en afrodescendientes colombianos, más aún cuando según datos del Observatorio de Discriminación Racial, el 61\% de los afrocolombianos no poseen el ingreso suficiente para suplir sus necesidades básicas de alimentación (14), un ejemplo de ello es la población de San Basilio de Palenque, patrimonio universal de la etnia africana en Colombia, que considera necesaria una mayor intervención por entes gubernamentales del 
sector salud en la implementación de programas de prevención y promoción de la salud.

Con respecto a la determinación de la hemoglobina, el índice primario de la serie eritroide, considerado en el laboratorio, el parámetro por excelencia de descarte para el estado anémico, se encontró normalidad dentro de los rangos de variabilidad biológica para el $100 \%$ de la población estudiada con una media de $15.1 \mathrm{~g} / \mathrm{dL}$, evidenciado con ello una población aparentemente sana y en estrecha relación con la altura sobre el nivel del mar.

Así mismo, el 24.6\% de la población estudiada se enmarca en el grupo del estado subclínico grado II de la deficiencia de hierro.Es decir hombres afrodescendientes valorados con hemoglobinas dentro de los rangos de variabilidad biológica normales, aparentemente sanosque demuestran con la valoración simultánea de los nuevos indicadores, el agotamiento de sus depósitos enmarcándose en estados carenciales plenamente instaurados.

Frente a la determinación de la Ferritina Sérica, el sTfR y el Indice Receptor Soluble de Transferrina, pruebas que en conjunto son más sensibles y especializadas, muestran un comportamiento similar a bajas alturas en las poblaciones estudiadas.

Por otra parte, al $85 \%$ de la población participante en este estudio se le debe continuar con los análisis con el fin de identificar las posibles causas que enmascaren la deficiencia de hierro, ya que es competencia del equipo médico buscar las causas incluídas las nutricionales, el poliparasitismo, las hemorragias, trastornos en la absorción intestinal del biometal, que conlleven a la presencia de los estadíos subclínicos. Posterior a ello es necesario instaurar medidas profilácticas y nutricionales que eviten la depleción de los depósitos de hierro a tiempo, antes que un sujeto desarrolle anemia ferropénica evitando las consecuencias por la ausencia del biometal.

Así mismo, los resultados encontrados demostraron una correlación estadísticamente significativa positiva entre el índice sTfR y una correlación estadísticamente significativa negativa con la FS, de lo cual se puede deducir que el índice se comporta directamente proporcional e inversamente proporcional con sus indicadores respectivamente.El índice sTfR-FSes una prueba altamente específica y sensible dado que potencializa los indicadores hematológicos.

Como hallazgo significativo, se encuentran los valores obtenidos del Índice Receptor soluble de tranferrina mayor a 2.3 con un mínimo de 2.4 y un máximo de 13.6, convirtiéndose en indicador importante para la continuidad del estudio en esta comunidad afrodescendiente.El uso índice sTfR-Log FS una medida de detección precoz que puede ser adoptada por el laboratorio de diagnóstico en hematología.

Finalmente, con este estudio se comprueba la efectividad de la aplicación del índice sTfR-FS como prueba alterna en la detección de las deficiencias de hierro en la población masculina se pueden instaurar planes de capacitación al recurso humano en salud con el fin de la adopción de este índice en la rutina médica.

Es importante anotar que este estudio servirá como base para posteriores investigaciones en población afrodescendiente con miras a mejorar la calidad de vida de estas comunidades, bajo el referente específico que el estado anémico ferropénico es de instauración lenta y progresiva, por lo tanto se debe tener cuidado en la detección de los casos y las familias en riesgo. De la misma forma, es importante precisar que el éxito del tratamiento depende de la determinación de la causa específica y aquellas que sean susceptibles de tratamiento tendrán una excelente evolución y recuperación positiva en los pacientes.

Como recomendaciones, sugerimos analizar de manera detallada los patrones gastronómicos de la comunidad para fortificar las dietas con hierro hemínico, así como las rutas de acceso para el ingreso de alimentos a la población, gestionar en colegios la consecución de meriendas que cumpla con todos los criterios de nutrición e implementar una serie de mecanismos que permitan solucionar esta situación.

\section{Agradecimientos}

Los autores agradecen a los 73 hombres afrodescendientes de San Basilio de Palenque, Institución Educativa Benkos Biohó; al Hospital Naval de Cartagena; a las directivas del programa de Bacteriologia de la Corporación Universitaria Rafael 
Núnez; a la Doctora Teresa Martínez, Epidemióloga; a la Doctora Liliana Díaz, Gerente de la casa comercial DIZAR Ltda., distribuidora y comercializadora; a la Doctora Elsa Cristina Soto Gómez de la Unidad Pediátrica Los Andes en Bogotá, D.C.

\section{Referencias}

1. Coy S, Castillo M, Mora I, Munevar A. Detección de deficiencias subclínicas de hierro a partir del índice receptor soluble de transferrina-ferritina en niños sanos de 1 a 10 ańos de edad residentes en alturas de 300 y 2600 msnm. Rev Nova 2009;7:43-51.

2. Doig K. Trastornos del metabolismo del hierro. En: Rodak B. Hematología: fundamentos y aplicaciones clínicas. 2a ed. Buenos Aires; Editorial médica Panamericana 2005: 213-225.

3. Beutler Ernest, West Carol. Hematologic differences between AfricanAmericans and whites: the roles of iron deficiency and thalassemia on hemoglobin levels and mean corpuscular volumen.Blood 2005;106:740-745.

4. Turgeon M. Hematología Clínica, Teoría y procedimientos. Colombia: Manual moderno; 2006.

5. Lozano J. Anemia ferropriva. En: Cuellar F, Falabella F. Fundamentos de Hematología. 6a ed. Colombia; Corporación para Investigaciones Biológicas; 2005:25-41.

6. Punnonen K, Rajama A, Irjala K. Serum Transferrin Receptor and Transferrin Receptor-Ferritin. Serum Transferrin Receptor and Its Ratio to Serum Ferritin in theDiagnosis of Iron Deficiency.Blood 1997:89:1052-1057
7. Skikne B, Flowers C, Cook J. Serum transferrin receptor: a quantitative measure of tissue iron deficiency. Blood 1990;75:1870-1876.

8. Suominen P, Punnonen K, Rajama A, Irjala K. Serum Transferrin Receptor and Transferrin Receptor-Ferritin Index Identify Healthy Subjects With Subclinical Iron Deficits. Blood 1998:92:2934-2939.

9. Coy S, Castillo M, Mora I, Munevar A, PeñaY. Características hematológicas de donantes de sangre de Bogotá, D.C., Colombia (2.600 m). RevMed 2007; 15:40-47.

10. Goyal R, DasR, BamberyP, GarewalG. Serum transferrin receptor-ferritin index shows concomitant iron deficiency anemia and anemia of chronic disease is common in patients with rheumatoid arthritis in north India. Indian J pathol microbio 2008;51:102-104.

11. Perry G, Byers T, Yip R. Margen SheldonIron Nutrition Does Not Account for the Hemoglobin Differences between Blacks and Whites. J. Nutr 1992;122: 1417-1424.

12. Malope, Babatyi, MacPhail Patrick, Alberts Marianne, Hiss Donavon. The ratio of serum transferrin receptor and serum ferritin in the diagnosis of iron status. Blood 2001:115;84-89.

13. Lopera J, et al. Estructura genética de la población actual del Palenque de San Basilio mediante el uso de retrotransposones de la familia humana TA (LINE1) trabajo de grado (pregrado en biología) Universidad Pontificia Javeriana. Facultad de Ciencias. Bolívar Colombia. REV. Salud Uninorte. 2004; 18:97.

14. Moro B. San Basilio de Palenque Frente a los Objetivos de Desarrollo del Milenioprograma de naciones unidas para el desarrollo Colombia; 2010. Disponible: http://www.pnud.org.co/img_upload/ 